\title{
Standardization of Herbs: An Essential Aspect for Drug Discovery \\ Pankaj Gupta*
}

School of Medical and Allied Sciences, KR Mangalam University, Sohna Road, Gurgaon, Haryana, India

*Corresponding author: Gupta P, School of Medical and Allied Sciences, KR Mangalam University, Sohna Road, Gurgaon, Haryana, India, Tel: +91-11-9818256122; Email: gupta.aiims@gmail.com

Received: March 14, 2018; Accepted: March 24, 2018; Published: April 02, 2018

Copyright: $\odot 2018$ Gupta P. This is an open-access article distributed under the terms of the Creative Commons Attribution License, which permits unrestricted use, distribution, and reproduction in any medium, provided the original author and source are credited.

\section{Editorial}

A number of approaches on drug discovery and development from traditional medicines have been practiced by scientists from world over for years together. The history of traditional medicines has helped in the development of several new molecules that have implications for new drug discovery and also act as precursors for providing new chemical entities. The values of natural products have been well studied and documented by our ancestors and since time immemorial, natural products have provided a vivid opportunity for researchers to use this documented knowledge for obtaining newer leads for drug discovery. Thereof, the secondary metabolites obtained from natural resources have been perceived as showing noticeable pharmacological actions with higher potency and lesser adverse effects making them good leads for further development. The new molecules in the form of secondary metabolites evolved in the nature through plants and animals and further research on these secondary metabolites with the help of new drug discovery tools is vital for the enhancement of human health. Most of the potent phytoconstituents of today including the aspirin to taxol have been derived from plants itself and beside these thousands of other phytoconstituents with therapeutic potentials would require more sophisticated techniques of rapid investigation initially for the purpose of standardization [1].

Standardization of crude herbal drugs is an inevitable part of the drug discovery process and refers to analyzing the quality and purity of the crude drugs before their further processing and remains a prerequisite step for the preparations of herbal formulations. It involves authentication of crude drugs, analyzing the quality and purity of the crude drugs via several means of evaluation such as organoleptic, microscopic, chemical, physical and biological means of evaluation. Among these evaluation techniques used for standardization of crude drugs, there are various methods that have been adopted since time such as morphological and sensory evaluation, chemical tests that have used for preliminary phytochemical screening, determination of ash value, extractive value, foreign matter etc. However, today's era involves the standardization of crude herbal drugs via fingerprinting technology wherein all the phytoconstituents present in the herb are enlisted in a sequential manner depending on the technique used for the purpose of fingerprinting. Fingerprinting of crude herbal drugs can be done in two ways. The first fingerprinting method involves usage of a chromatographic technique such as high-performance liquid chromatography (HPLC), gas chromatography (GC) etc. or a chromatographic technique coupled with a analytical techniques such as liquid chromatography coupled with two mass spectrometers (LCMS/MS) or GC coupled with mass spectroscopy (GC-MS) etc. The second methods for fingerprinting involves DNA fingerprinting technique wherein the DNA analysis of the crude drug has been used for its authentication and therefore act as an important tool for herbal drug standardization [2].

The problem of quality assurance in case of crude herbal drugs can be resolved to a great extent with the help of fingerprinting techniques as the quality grade could be determined successfully using the fingerprint spectra of crude herbal drugs [3]. The variation determination of common peaks, noticeable in the chromatographic fingerprint spectra could provide qualitative as well as quantitative information related to the phytoconstituents present in the crude herbal drugs. Pattern recognition of the chromatographic fingerprint spectra can be used for discrimination of different kind of sample of crude herbal drugs. Thus, chromatographic fingerprinting serves as a promising quality control tool for crude herbal drugs. DNA fingerprinting is another technique that has proven to be a promising tool for the standardization and quality control of crude herbal drugs.

\section{References}

1. Adams JD, Flora KP, Goldspiel BR, Wilson JW, Arbuck SG, et al. (1993) Taxol: A history of pharmaceutical development and current pharmaceutical concerns. J Natl Cancer Inst Monogr 15: 141-147.

2. Gupta P (2015) DNA and LC-MS/MS finger printings as novel tools for the standardization of crude herbal drugs. Nat Prod Chem Res 3: 3 .

3. Kulkarni KM, Patil LS, Khanvilkar VV, Kadam VJ (2014) Fingerprinting techniques in herbal standardization. Indo American J Pharm Res 4: 1049-1062. 